\title{
Planetary Accretion Rates: Analytical Derivation
}

\author{
Richard Greenberg and William F. Bottke \\ Lunar and Planetary Laboratory, University of Arizona, Tucson, Arizona 85721
}

AND

Andrea Carusi and G. B. Valsecchi

IAS Reparto Planetologia. V. Universita II, 00185 Rome, Italy

Received February 19, 1991: revised July 31, 1991

\begin{abstract}
Analytic formulae for estimating planetary accretion rates have long been available for conditions where random motion of planetesimals controls velocities relative to a growing planet. Recent Monte Carlo studies have explored a wider range of conditions, but gave impact rates only for the specific conditions under which those numerical experiments were performed. Now analytical formulae, which are valid even when keplerian shear controls the approach velocity, and which accurately reproduce the Monte Carlo results, give accretion rates over a wide range of conditions relevant to the problem of planet formation. 1991 Acadernic Press. Inc.
\end{abstract}

\section{INTRODUCTION}

The dynamics of encounters of planetesimals with growing planetary embryos have been traditionally modeled using a two-body approximation (Öpik 1951, Safronov 1969). Greenberg et al. (1978a,b) developed a numerical simulation of a population of collisionally interacting planetesimals, which also used the two-body model to estimate impact probabilities and the results of close encounters. That simulation used a "particle-in-a-box" treatment, in which the system is followed in a frame moving around the Sun with keplerian circular velocity. In that algorithm, random velocities, corresponding to the eccentricities $e$ and inclinations $i$ of individual particles. were assumed to be great enough that mutual approaches are primarily governed by the random motion, rather than by keplerian shear, which would dominate if $e$ and $i$ were sufficiently small.

One result of the simulations by Greenberg et al. which contradicted earlier models, was that a few planetary embryos could quickly grow to nearly $1000 \mathrm{~km}$ in diameter while most of the mass of the system remained in much smaller bodies (near the size of the original planetesimals $-1 \mathrm{~km}$ ). That mass distribution caused the random veloci- ties of all the bodies to remain small. This result was consistent with Safronov's (1969) demonstration that random velocities would be comparable to the escape velocities of the dominant bodies, except that Safronov had assumed that the largest bodies would always be dominant. With relative velocities remaining small, the results were quite different from Safronov's: The gravitational cross-sections of the largest bodies became quite large, setting the stage for accretional "runaway growth." This general character of evolution of the system through this stage has been confirmed by various more recent numerical simulations (e.g., Wetherill and Stewart 1989).

Greenberg et al. $(1978 \mathrm{a}, \mathrm{b})$ noted, however, that the low relative velocities make their algorithm questionable as the size of the largest body (embryo) approaches $\sim 1000$ $\mathrm{km}$. The reason for their caution was that, beyond that size, the gravitational cross-section is larger than the verti$c a l$ and radial excursions due to the random ( $i$ and $e$ ) part of planetesimals' motion. In effect, the approach of planetesimals to the embryo is governed by keplerian shear (with $e$ and $i$ negligible), rather than by the random motion. Thus, although "incipient runaway growth" is underway at this point, the character of further evolution of the swarm remains a matter of speculation (see review by Greenberg 1989).

Motivated in part by this difficulty, Wetherill and Cox (1984, 1985) performed a large number of numerical integrations of the motions of test particles approaching hypothetical planets. They found that when the ratio $V / V_{\text {c }}$ (random velocity of test particle to escape velocity of planet) is less than $\sim 0.1$, the impact probability diverges from the prediction of the "particle-in-a-box" type of calculation. They suggested that the discrepancy involved some sort of breakdown of the two-body model at encounter.

However, from a detailed investigation of the behavior 
of particles during such encounters, Greenberg et al. (1988a) concluded that generally, aside from some statistically unimportant pathological cases, behavior during close encounters follows hyperbolic two-body motion even in the low $V / V_{\mathrm{e}}$ regime where Wetherill and Cox suggested it broke down. Greenberg et al. (1988a) explained the transition at $V / V_{\mathrm{e}} \sim 0.1$ by the change from (A) a regime in which random motion dominated approach toward close encounter to (B) a regime in which keplerian shear dominated. In other words, the transition noted by Wetherill and Cox was the same as the limit of validity predicted by Greenberg et al. (1978a).

The understanding that the two-body approximation was valid even in the regime of low $V / V_{\mathrm{e}}$, where keplerian shear dominates, allowed Greenberg et al. (1988b) to construct analytic formulae that agreed with and explained the impact rates found in the numerical experiments by Wetherill and Cox. In this paper, we show how those analytic formulae were derived and show the comparison with the numerical results of Wetherill and Cox.

Moreover, two other research groups have recently performed numerical experiments similar to those of Wetherill and Cox. Some of those experiments include results in a third regime of extremely small values of $V / V_{\mathrm{e}}$. The analytic formulae derived here (Section II) include expressions for criteria for the transitions between the various regimes, and are in excellent agreement with the results of all these numerical experiments. The predictive success of our formulae indicates that we now have a reasonably good understanding of some of the fundamental processes controlling impact rates.

\section{DERIVATION OF ACCRETION-RATE FORMULAE}

Protoplanet-planetesimal collision rates within the solar nebula can be categorized into three primary regimes. Conditions in which random motion is the dominant mechanism for bringing material within the sphere of influence of the protoplanet is referred to here as Regime A. Conditions under which keplerian shear (i.e., bodies closer to the Sun move faster than ones further away) is the dominant mechanism for bringing material is referred to as Regime B. If keplerian shear dominates but the planetesimal swarm is nearly flat, the system is in Regime C. In Regime $C$ the dynamics are essentially two-dimensional (in contrast to $\mathrm{A}$ and $\mathrm{B}$ where behavior requires a threedimensional model) as the material enters the protoplanet's sphere of influence only from the plane of the disk.

In accord with the results of Greenberg et al. (1988a) we derive formulae for accretion rates in these regimes by assuming that two-body behavior can give a good approximation of encounters, even at low velocity, if one properly describes the approach trajectory.
In the following sections we derive accretion rate formulae in a way that demonstrates the essential physics. We then compare the rates given by our formulae with the rates found in the various sets of results of numerical experiments now in the literature. This comparison is straightforward in principle, but requires considerable care to ensure that the various choices of scaling adopted in the literature are correctly taken into account. Although our objective was to derive order-of-magnitude expressions that reveal the important functional dependencies, we find that our analytic expressions closely match the numerical results, both in values of accretion rates and in the functional dependencies on the controlling parameters.

\section{II.A Dominance by Random Motion (Regime A)}

As long as the random velocities $V$ of planetesimals are sufficiently large, keplerian behavior is unimportant. Random velocity is the radial and vertical motion relative to circular, coplanar orbits, which corresponds to orbital eccentricity $e$ and inclination $i$. Thus, traditionally the dynamics of approach and encounter are modeled by twobody behavior in a "gas" of particles. The impact rate is roughly the product of the target protoplanet's crosssectional area, and the flux of particles upon it. The flux is $V$ times the density (number/volume) of the particles in the disk. The density is given by the surface density $\sigma$ divided by the thickness of the swarm of small particles. The thickness is given roughly by $2 a i$, where $a$ is the distance from the Sun and $i$ is the orbital inclination.

Thus, the number of particles per unit time hitting the protoplanet is

$$
\text { Impact rate } \sim \pi R^{2}\left(\frac{V \sigma}{2 a i}\right) .
$$

In (1) we have assumed that $V$ is so large that, not only does it dominate over keplerian shear in controlling the approach, but it prevents the planet's gravity from enhancing the target cross-section. The target area is thus taken to be $A=\pi R^{2}$ in deriving (1). Also,

$$
V \sim n a \sqrt{e^{2}+i^{2}},
$$

where $a$ is the distance from the Sun, $n$ is the mean motion, and $n a$ is the planet's circular orbital velocity around the Sun.

In the more general case (still in Regime A) where the cross-section is enhanced by the planet's gravity in accordance with two-body scattering:

$$
A \sim \pi R^{2}\left(1+\frac{V_{\mathrm{e}}^{2}}{V^{2}}\right)
$$

Therefore, 


$$
\text { Impact rate } \sim \pi R^{2}\left(1+\frac{V_{\mathrm{e}}^{2}}{V^{2}}\right)\left(\frac{V \sigma}{2 a i}\right)
$$

where again $V$ is given by (2).

To the level of precision appropriate for many considerations of planet formation or of other problems in planetary collision dynamics, these approximate equations can be, and often have been, quite properly used without further refinement (e.g., Wetherill and Cox 1984, 1985).

Moreover, these equations can be further simplified, because in most collisionally evolved systems $e \sim 2 i$. This approximate relation was found in numerical simulations by Greenberg et al. (1978a,b) and by Wetherill (1980). Consequently Stewart and Wetherill (1988) assumed that it holds exactly and Wetherill and Cox $(1984,1985)$ restricted their studies to the case $e=2 i$. The $e / i$ ratio is controlled by energy partitioning, where $e$ determines the random velocity in the two in-plane dimensions (not equally) and $i$ controls the random velocity in the one normal-to-the-plane dimension. These relations only apply to average values of $e$ and $i$; significant components of the population may in fact lie in other parts of $e, i$ space. In a system where $e \sim 2 i$, we would have $i-V / n a$, so the impact-rate formulae are greatly simplified: $V$ and $i$ can be eliminated from (1), and $i$ can be eliminated from (4).

In this paper the analysis is not restricted to any specific ratio of $e / i$, because our objective is to find more general formulae that can be compared with the results of Monte Carlo experiments, and thereby explain and generalize them. As in the Monte Carlo experiments, the swarm of planetesimals is assumed to be characterized by a single value of $e$ and one of $i$. Investigation of the effect of various distributions of $e$ and $i$ is beyond our objective here. As demonstrated in Appendix A, the above equations governing impact rates need only be modified slightly to become quite accurate. Accordingly, we adopt the form

$$
\text { Impact rate }=\frac{5}{8} \pi R^{2}\left(1+\frac{V_{c}^{2}}{V^{2}}\right)\left(\frac{V \sigma}{2 a i}\right)
$$

where $V$ is given by Safronov's (1969) expression

$$
V=n a \sqrt{\frac{5}{8} e^{2}+i^{2}} .
$$

Equations (5) and (6) retain the same simple form and physical interpretation as the approximations (4) and (2). Note that these equations give the impact rate as a function of the characteristic orbital eccentricities and inclinations of the planetesimals, $e$ and $i$. In the following sections we derive analogous expressions for conditions where the assumptions of Regime A are not valid.

\section{II.B Dominance by Keplerian Shear Motion (Regime B)}

1. Transition from Regime $A$ to Regime $B$. For larger values of $V_{\mathrm{e}} / V$, approach dynamics begin to be dominated by keplerian shear rather than random motion. If all planetesimals were on nearly circular and coplanar orbits (very small $V$ ), they would pass by the planet due to their differential keplerian velocities. As they passed, the planet's gravitational pull would stimulate eccentric motion, which might allow orbit crossing and, ultimately, impacts with the planet. Contrary to Regime $\mathrm{A}$, this process is not controlled by the initial random motion.

The keplerian feeding zone is the region over which particles even on initially nearly circular orbits are perturbed onto planet-crossing orbits. Now suppose the planetesimals in this feeding zone have some initial random motion, with radial excursion $e a$. If $e a$ is significantly smaller than the range of the feeding zone $\Delta a$, then the bulk of the planetesimals reaching the planet's orbit do so despite their initially small eccentricities. Thus $\Delta a \sim e a$ defines the limit of validity of Regime $A$ and the transition into Regime B.

The half-width $\Delta a$ of the feeding zone can be estimated in various ways. For example, consider a planetesimal on a circular orbit (radius $a+\Delta a$ ) going past a planet (orbital radius $a$ ). The dominant effect of the planet occurs while the planetesimal is within a longitudinal distance $\sim \Delta a$ with respect to the planet. That condition lasts for a time $\sim 2 \Delta a / \frac{1}{2} n \Delta a=4 / n$ according to Kepler's laws. The change in velocity required to put the planetesimal on a planet crossing orbit is about $n \Delta a$; the change due to the pull of the planet is the acceleration $G M_{\mathrm{p}} /(\Delta a)^{2}$ times the duration $4 / n$. Equating these two expressions for the change in velocity, and using Kepler's third law, yields the half-width of the feeding zone,

$$
\Delta a \approx a\left(\frac{4 M_{\mathrm{p}}}{M_{\mathrm{C}}}\right)^{1 / 3}=2.3 R_{\mathrm{H}},
$$

where $R_{\mathrm{H}}$ is the radius of the Hill sphere derived from Hill's equations (Hill 1898), which is the approximate size of the planet's potential well in the rotating reference frame of the restricted three-body problem:

$$
R_{\mathrm{H}} \equiv a\left(\frac{M_{\mathrm{p}}}{3 M_{3}}\right)^{1 / 3}
$$

Numerical studies (e.g., Dermott and Murray 1981, Nishida 1983, Petit and Henon 1986, Burns et al. 1989) of swarms with test particles on circular orbits show maximum feeding distances consistent with (7), although the agreement is more precise if we adopt 


$$
\Delta a=2.5 R_{\mathrm{H}} .
$$

Equating this expression to $e a$ gives the criterion for transition from Regime A to B:

$$
e=2.5\left(\frac{M_{\mathrm{p}}}{3 M_{\odot}}\right)^{1 / 3}
$$

Equation (4) for impact rates in Regime $A$ is a function of $e$ and $i$; Eq. (10) gives the boundary of its validity in $(e, i)$ space. This transition is gradual and occurs over an order-of-magnitude in $e$ values. For much larger values of $e$, a system is in the regime of random velocity control (Regime A); for much smaller values of $e$, a system is in the regime of keplerian shear dominance, or Regime $B$.

If $e \sim 2 i$, as in any real collisionally developed system, the left side of (10) is proportional to the random velocity $V$. The right side is proportional to the planet's escape velocity $V_{\mathrm{e}}$. Thus in this restricted part of $(e, i)$ space, Eq. (10) could be expressed as a limiting value of $V / V_{c}$, below which the impact-rate equations of Regime $A$ are invalid.

More specifically, consider the case $e=2 i$. With Kepler's third law and our adopted form of $V$, Eq. (10) yields

$$
\left[\frac{V}{V_{\mathrm{c}}}\right]_{\mathrm{A}-\mathrm{to}-\mathrm{B}}=0.9 G^{-1 / 6} \rho_{\mathrm{p}}^{-1 / 6} n^{1 / 3}
$$

For a rocky planet $\left(\rho_{\mathrm{p}} \sim 4 \mathrm{~g} \mathrm{~cm}^{-3}\right)$ in orbit about the Sun at $1 \mathrm{AU}$, the right side is about 0.1 , which explains why the Monte Carlo results of Wetherill and Cox diverged from the impact-rate formulae in Section II.A for smaller $V / V_{\mathrm{e}}$. Note that according to (11) the critical value of $V / V_{\mathrm{e}}$ is not necessarily near 0.1 for all cases. It would be different if $M_{\odot}, a$, or $\rho_{\mathrm{p}}$ were changed considerably, but the dependence on these parameters is quite weak. Section III contains further detailed quantitative comparisons between our analytic formulae and various Monte Carlo studies.

2. Impact rate in Regime $B$. The impact rate in this regime depends in part on the flow rate of planetesimals into the vicinity of the planet, due to keplerian shear. This "feeding flow" depends on the width of the feeding zone, the shear velocity, and the surface number density. The full width of the feeding zone is $2 \Delta a$ (a distance $\Delta a$ both inward and outward) as shown in Fig. 1, which shows the flow in a reference frame centered on the planet and rotating at the rate of the planet's orbital motion.

Planetesimals on circular orbits very close to that of the planet have their orbital energies changed and their relative angular velocities reversed before they pass by

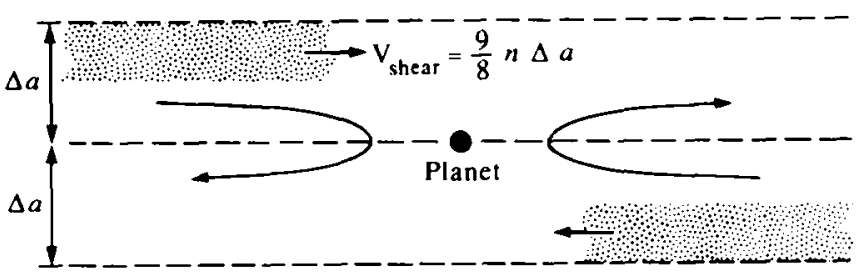

FIG. 1. A planetary feeding zone, shown looking down on the planet's orbit plane in a reference frame moving with the planet. Planetesimals with semimajor axes as far as $\Delta a$ from that of the planet may be diverted onto orbits that cross the planet's orbit. Planetesimals from within the core of this zone have their orbital energy changed enough that their relative motion is reversed and they move away from the planet (horseshoe orbits). The other bodies approach the planet with an average velocity, due to keplerian shear, of $\frac{2}{8} n \mathrm{~J} a$.

the planet, i.e., they are on "horseshoe orbits." This process prevents collisions by planetesimals on initial orbits very close to the planet's. This critical range can readily be shown analytically to be about one Hill radius (e.g., Goldreich and Tremaine 1982). Thus in the following calculations we assume that the inner core (the part closest to the planet) of the feeding zone is a region of avoidance which extends half way from the orbit of the planet to the edges of the feeding zone (Fig. 1). In our approximation, the total width of the remaining feeding flow is $\Delta a$.

In fact numerical studies (e.g., Dermott and Murray 1981, Nishida 1983, Petit and Henon 1986, Burns et al. 1989 ) indicate that this avoidance zone extends to 1.75 $R_{\mathrm{H}}$, or 0.7 of the full width of the feeding zone. Adopting the more precise width instead of 0.5 would change the impact rates derived below by only $15 \%$, well within the uncertainty of the calculation.

The flow speed in the rotating reference frame for particles on circular orbits at either extreme edge of the feeding zone is $\frac{3}{2} n \Delta a$, according to Kepler's third law. The average feeding flow speed, without the inner core of horseshoe orbits, is $\frac{3}{4}$ as large, or $\frac{9}{8} n \Delta a$.

Thus the feeding flow (number of particles/time) approaching the planet in this way is

$$
\text { Flow rate }=\left(\frac{9}{8} n \Delta a\right) \sigma \Delta a
$$

Most of the planetesimals in this feeding flow not only have their trajectories bent toward the planet's orbit, but they also enter the Hill sphere (Fig. 2). This expectation is confirmed by the various numerical integrations (referenced above) of initially circular orbits.

Moreover, nearly all of these bodies enter the Tisserand sphere of influence as well. The Tisserand sphere is defined as the region around a planet where the motion of small particles is better approximated by two-body motion 


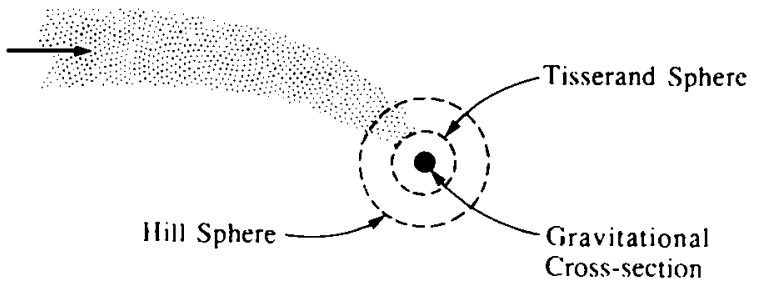

FIG. 2. Nearly all the material that approaches the planet due to keplerian shear (excluding particles on horseshoe orbits), is diverted into the Tisserand sphere and the larger Hill sphere around the planet. according to numerical integration of originally circular orbits. Here we show schematically only one of the two approaching bands of flow from Fig. 1

relative to the planet than by two-body motion relative to the Sun. The radius of the Tisserand sphere (Roy 1988) is

$$
R_{\mathrm{T}}=a\left(\frac{M_{\mathrm{p}}}{M_{\Theta}}\right)^{2 / 5}
$$

Note that $R_{\mathrm{T}}$ is about $90 \%$ of $\boldsymbol{R}_{\mathrm{H}}$ for Jupiter, or $50 \%$ of $\boldsymbol{R}_{\mathrm{H}}$ for a Moon-sized planet. It is the same order of magnitude (albeit somewhat smaller) as $R_{\mathrm{H}}$ for most cases of interest in planetary studies.

For purposes of our analytical estimate of impact rates, we assume that once a planetesimal gets close enough to the planet, its trajectory can be approximated by twobody motion governed by the planet. We adopt the Tisserand sphere as the region in which this estimate is valid. (An anonymous referee correctly notes that the dynamics of this problem scales with the size of the Hill sphere. However, for invoking two-body behavior as an approximation of part of the motion, the Tisserand sphere is the relevant boundary. We could have adopted the Hill sphere with minimal quantitative consequences, to the desired degree of precision.)

In order to invoke two-body motion within the Tisserand sphere, we need to estimate the velocity at which particles enter that region. Roughly speaking, the velocity must be $\sim n \Delta a$, because that is the magnitude of the induced eccentric motion that brings the particles onto the planet approaching trajectories.

We can refine that estimate by using the Jacobi integral of the restricted three-body problem (Danby 1988). Suppose a particle is initially on a circular orbit, $180^{\circ}$ from the orbital position of the planet, with orbital radius greater than the planet's by an amount $\frac{3}{4} \Delta a$ (i.e., in the middle of one of the zones of feeding flow). If we evaluate the Jacobi integral at that initial condition, and equate it to its value at a point entering the Tisserand sphere at a point $R_{\mathrm{T}}$ from the planet in the direction opposite to the sun, we find

$$
\begin{gathered}
V_{\mathrm{T}} \approx 0.58\left(2 \mu{ }^{1: 1.5}-1.27\right)^{1.2} \Delta a . \\
\text { For } \mu=10^{\circ} . \\
V_{\mathrm{T}} \approx 1 . \ln \Delta a .
\end{gathered}
$$

To the level of precision of our analysis, this result does not differ significantly for initial orbits elsewhere in the feeding zone, or for arrival at other points on the Tisserand sphere. Similarly, although this velocity is in the rotating reference frame of the restricted three-body problem, conversion to the inertial frame would involve an insignificant correction. This result is fairly insensitive to the value of $\mu$ over the range of values likely to be relevant in the planet formation problem. Thus, we adopt (14a) as the typical speed of a body entering the Tisserand sphere.

Given $V_{\mathrm{T}}$, the two-body gravitational cross-sectional area of the planet within the Tisserand sphere is

$$
A_{\mathrm{G}}=\pi R_{\mathrm{G}}^{2}=\pi R^{2}\left(1+\frac{V_{\mathrm{c}}^{2}}{V_{\mathrm{\Gamma}}^{2}}\right) .
$$

As shown in Appendix B, unless the planet's orbit is nearly as small as the Sun or its density is much less than the Sun's,

$$
R \ll R_{\mathrm{G}} \ll R_{\mathrm{T}} \sim R_{\mathrm{H}} .
$$

Here in Regime B, we assume that the vertical thickness $2 a i$ of the swarm of planetesimals is greater than the diameter $2 R_{\mathrm{G}}$ of the gravitational cross-section (as in Fig. 3), otherwise the system is in the very low $i$ (essentially twodimensional) Regime $C$, considered in the next section.

Although $a i>R_{G}$, it is usually less than the radius of the Tisserand sphere $\boldsymbol{R}_{\mathrm{T}}$, as shown in Fig. 3 for the following reason: In a collisionally evolved system, $e$ and $i$ would be comparable in value. In Regime B, except very near

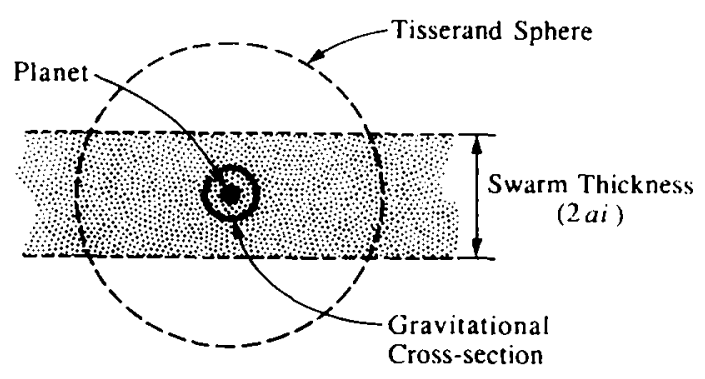

FIG. 3. An "edge-on" view of the planetesimal swarm and the planet from the planet's orbital plane, showing for Regime $B$ the relative scale of the swarm thickness, the Tisserand sphere, the physical size of the planet. and the gravitational cross-section. For Regime C, the swarm is thinner than the Tisserand sphere. 
the transition zone to Regime $\mathrm{A}, e a \ll R_{\mathrm{T}}$, so we expect $a i \ll R_{\mathrm{T}}$ as well. However, that assumption is not necessary in the following derivation.

Once a planetesimal enters the Tisserand sphere, the probability of impacting the planet is the target crosssection $A_{\mathrm{G}}$ from (15) divided by the cross-sectional area of the flow (Fig. 3):

$$
\text { Impact probability }=\frac{\pi R^{2}\left(1+\frac{V_{\mathrm{e}}^{2}}{V_{\mathrm{T}}^{2}}\right)}{\left(2 R_{\mathrm{T}}\right)(2 a i)} .
$$

(The estimate may be slightly too low, because the particles in the swarm encountering the Tisserand and Hill spheres tend to be moving more nearly perpendicular to the sphere than a random swarm, according to inspection of various published numerical integrations, e.g., Nishida's. It is plausible that this effect roughly offsets effects of other estimates above, but in any case they are negligible to our targeted level of precision.) The impact rate is the flow rate (12) times the impact probability:

$$
\text { Impact rate }=\pi R^{2}\left(1+\frac{V_{\mathrm{c}}^{2}}{V_{\mathrm{T}}^{2}}\right)\left(\frac{(\sigma \Delta a)\left(\frac{9}{8} n \Delta a\right)}{\left(2 R_{\mathrm{T}}\right)(2 a i)}\right),
$$

where $\Delta a, R_{\mathrm{T}}$, and $V_{\mathrm{T}}$ are given by Eqs. (9), (13), and (14). Note that for a given planetary mass (relative to the Sun's) and radius (relative to $a$ ), the impact rate (18) varies inversely as $i$ and is independent of $e$. In comparison, in Regime A, the impact rate (5) depends on $e$ and $i$.

$\mathrm{S}$. Ida (private communication) notes that, in the extreme case where $i \gg e$ and $i \gg \Delta a$, the above derivation of the impact rate for Regime $B$ would not be valid for the following reason: the change in the in-plane velocity of a particle on an initially nearly circular orbit as it moves past the planet would be much reduced if the out-ofplane motion far exceeded $\Delta a$. Thus for $i \gg 2.5 R_{\mathrm{H}} / a$, it is expected that accretion rates might be considerably smaller than given by (18).

\section{II.C Keplerian Shear Dominance in a Very Thin Disk (Regime C)}

1. Transition from Regime $B$ to Regime $C$. In evaluating the impact rate for Regime $B$, we assumed that the protoplanet's gravitational diameter $R_{\mathrm{G}}$ [defined by Eq. (15)] was smaller than the vertical thickness $2 a i$ of the planetesimal swarm (as in Fig. 3). This assumption doesn't always hold. If $a i<R_{\mathrm{G}}$, the system is effectively twodimensional (Regime $\mathrm{C}$ ). By this definition, the transition from Regime $B$ to $C$ occurs when

$$
a i \approx R_{\mathrm{G}} .
$$

Equation (19) represents a boundary in $(e, i)$ space between Regimes B and C, just as (10) represents a boundary between $A$ and $B$. The transition between regimes is gradual across each boundary, rather than abrupt.

The value of $i$ at the B-to-C transition (19) is much smaller than the value of $e$ at the A-to-B transition (10), according to the following comparison. Using (B6) from Appendix B, (19) yields

$$
i=0.9\left(\frac{R}{R_{\mathrm{H}}}\right)^{1 / 2} \frac{R_{\mathrm{H}}}{a}
$$

at the B-to-C transition. In comparison, (10) yields

$$
e=2.5\left(\frac{R_{\mathrm{H}}}{a}\right)
$$

at the A-to-B transition. The ratio of (20) to (21) is < $\left(R / R_{\mathrm{H}}\right)^{1 / 2}$ which is $\ll 1$ (Appendix $\mathrm{B}$ ).

In the interesting case $e=2 i,(21)$ is equivalent to the critical value $\left[V / V_{\mathrm{e}}\right]_{\mathrm{A}-\mathrm{to}-\mathrm{B}}$ in (11). Similarly,

$$
\left[\frac{V}{V_{\mathrm{e}}}\right]_{\mathrm{B}-\mathrm{lo}-\mathrm{C}}=0.6 G^{-1 / 3} \rho_{\mathrm{p}}^{-1 / 3} n^{2 / 3} .
$$

Thus, we confirm that this transition occurs at a much smaller value of $V / V_{\mathrm{e}}$ than the A-to-B transition. We also find the remarkable result

$$
\left[\frac{V}{V_{e}}\right]_{B-10-C}=0.75\left[\frac{V}{V_{e}}\right]_{A-10-B}^{2}
$$

independent of the choice of parameters.

Note that even in Regime C, the approach of planetesimals to the planet is governed by keplerian shear, just as in Regime $\mathrm{B}$.

2. Impact rate in Regime $C$. In this regime the feeding flow rate is identical to Regime B. However, the impact probability now becomes the gravitational cross-section diameter divided by the width of the flow [cf. Eq. (17)]:

$$
\text { Impact probability }=\frac{2 R\left(1+\frac{V_{\mathrm{e}}^{2}}{V_{\mathrm{T}}^{2}}\right)^{1 / 2}}{2 R_{\mathrm{T}}}
$$

As in Regime B, the impact rate is simply the feeding flow rate times the impact probability [cf. Eq. (17)]

$$
\text { Impact rate }=R\left(1+\frac{V_{\mathrm{e}}^{2}}{V_{\mathrm{T}}^{2}}\right)^{1 / 2} \frac{(\sigma \Delta a)\left(\frac{9}{8} n \Delta a\right)}{R_{\mathrm{T}}} .
$$


This expression is independent of $e$ and $i$, and thus of $V$, as will be evident in our comparison with impact rates from Monte Carlo numerical experiments (Section III).

\section{COMPARISON WITH MONTE CARLO NUMERICAL EXPERIMENTS}

Several studies of impact rates for small particles encountering hypothetical planets have been performed in recent years using a Monte Carlo approach in which swarms of randomly selected particle orbits are numerically integrated to develop impact statistics. In Section III we compare the impact rates given by our analytical theory with those various Monte Carlo results.

\section{III.A. Experiments by Wetherill and Cox}

Of the extensive experiments by Wetherill and Cox $(1984,1985)$, the set most relevant to planet growth is that where $e=2 i$ (Fig. 9 of their 1985 paper). Their other cases have $i=0$ and are not directly relevant here. In that set of experiments $e$ is held constant at 0.00447 and the planet's density is fixed at $4.04 \mathrm{~g} \mathrm{~cm}^{-3}$. Experiments were run for a variety of assumed planetary radii ranging from about 800 to $8000 \mathrm{~km}$. In Fig. 4 we reproduce their results.

On the horizontal axis, $V_{\mathrm{w}}$ is the random velocity defined by the approximate form adopted by Wetherill and Cox [our Eq. (2)] rather than the more refined form of $V$ that we adopt in Eq. (6). The variation in $V_{\mathrm{e}} / V_{\mathrm{w}}$ on this axis is strictly due to changes in the planetary radius; $V_{w}$ is constant throughout because both $e$ and $i$ were fixed in these experiments. On the vertical scale, the "Gravitational Enhancement Factor, $F_{g}$ " was defined as the ratio of the impact rate to the nongravitational impact rate, which Wetherill and Cox determined by running their Monte Carlo experiments for a "standard" reference case with $V_{\mathrm{e}} / V_{\mathrm{w}}$ so small that gravitational enhancement over the geometrical cross-section is negligible.

The solid line in Fig. 4 shows the accretion rates given by our formulae for Regimes $A$ and $B$, plotted on the same scaled axes. For purposes of illustration, the transition between regimes is shown by an abrupt discontinuity at the value of $V_{\mathrm{e}} / V$ (scaled to the definition of $V_{\mathrm{e}} / V_{\mathrm{u}}$ ) given by (10).

Note the excellent agreement between our theory and the experimental results, which is even more striking when one recalls that our theory actually predicts a gradual transition between regimes over an order-of-magnitude in $V_{\mathrm{e}} / V$. Our analytical theory clearly reproduces all the features noted by Wetherill and Cox, including the extra enhancement over the extrapolated two-body formula for a limited range of $V_{\mathrm{e}} / V_{\mathrm{w}}$ values (Wetherill and Cox call this enhancement "anomalous gravitational focusing"), and the change in slope for larger values of $V_{\mathrm{e}} / V_{\mathrm{w}}$.

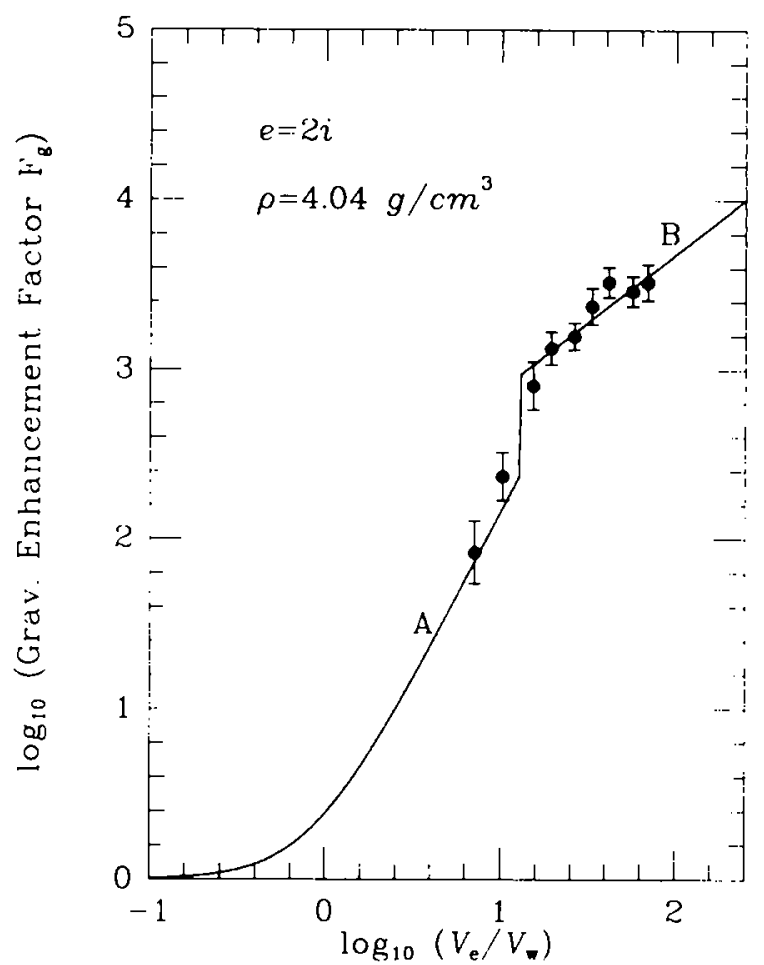

FIG. 4. Results of Monte Carlo numerical experiments of Wetherill and $\operatorname{Cox}(1985$, data points with error bars are from their Fig. 9), compared with our analytical results (solid curve) plotted on the same scale. $F_{\alpha}$ is the impact rate scaled to the case where random velocities are so large that geometric cross-section controls the rate. $V_{w}$ [defined precisely in terms of $e$ and $i$ by Eq. (2)] is the approximate random velocity adopted by Wetherill and Cox and was held constant in all the experiments represented here: $V_{\mathrm{c}}$ was varied to give change along the abscissa. Our theoretical transition from Regime A (random motion dominant) to Regime B (keplerian shear dominant) is shown by an abrupt discontinuity. although our model actually predicts a gradual transition over an order of magnitude in $V_{\mathrm{c}} / V_{\mathrm{w}}$. Agreement between theory and experiment is excellent.

\section{III.B. Experiments by Greenzweig and Lissauer}

Greenzweig and Lissauer (1990) performed similar Monte Carlo experiments. Their Fig. 8 shows results in a format very similar to that introduced by Wetherill and Cox (Fig. 10). Those results are reproduced as the data points shown in our Fig. 5. The data are plotted with $V \equiv$ $V_{\text {(il. }}$ [Eq. (A8)] which is the definition adopted by Greenzweig and Lissauer; recall however that our adopted definition $V \equiv V_{\text {Saf }}$ [Eqs. (6) or (A10)] is equal to $V_{\text {Gi }}$ within a few percent as discussed in Appendix A. The Gravitational Enhancement Factor is the accretion rate scaled to the geometric rate adopted by Greenzweig and Lissauer, which also is very nearly identical to the geometric rate that we have adopted $\mid \mathrm{Eq}$. (A12) in Appen$\operatorname{dix} \mathrm{A}]$.

In these experiments, $V_{\mathrm{v}} / V$ was varied by changing $V$ (through $e$ and $i$ ), rather than by changing $V_{\mathrm{e}}$ (through $R$ ) 

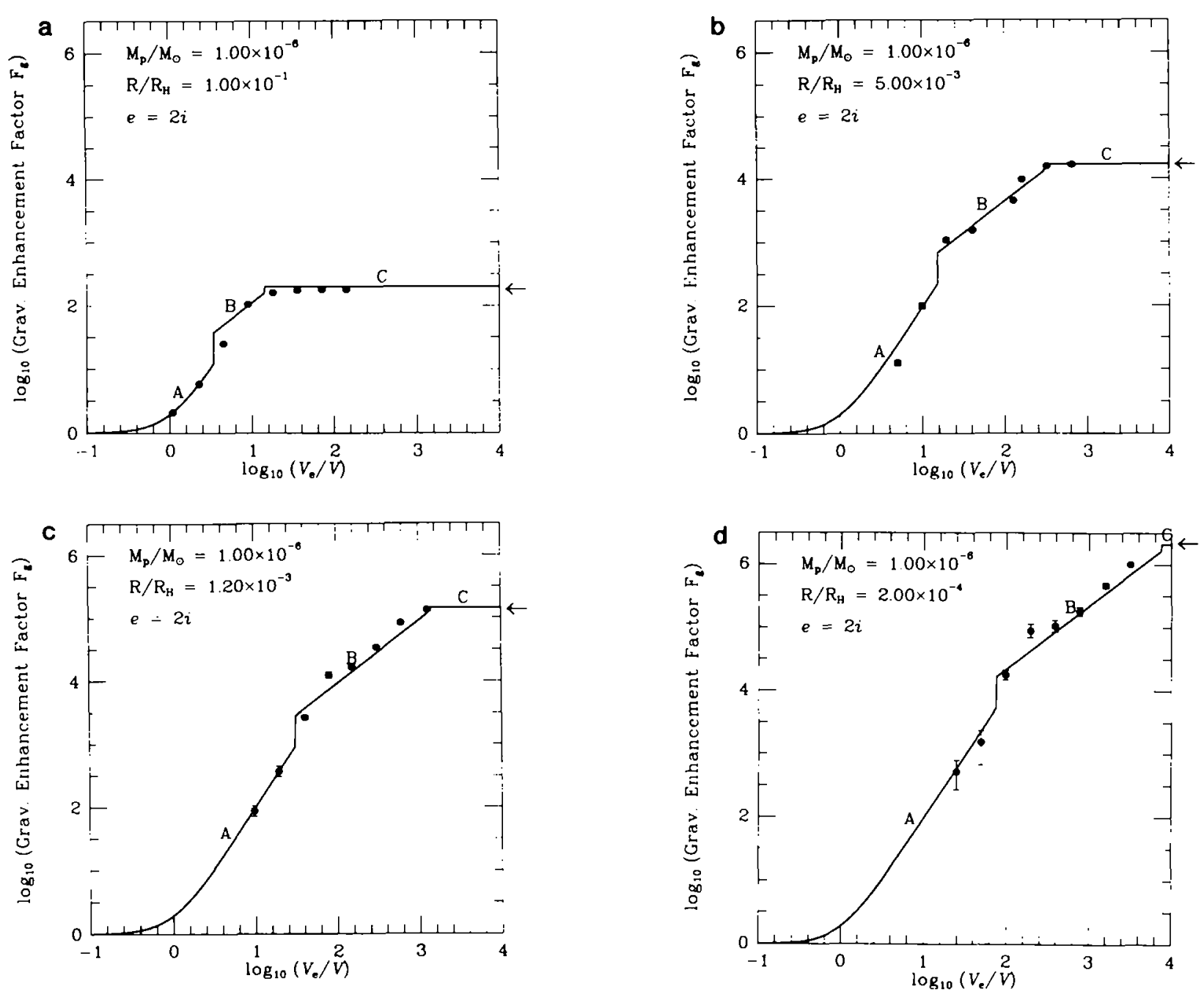

FIG. 5. Results of Monte Carlo numerical experiments of Greenzweig and Lissauer (1990, their Fig. 8) showing agreement with our analytical theory (solid curves). Their results for $V=0$ (off-scale in this format) is indicated by an arrow at the right. In the four cases (a-d), the ratios $e / i$ and $M_{\mathrm{p}} / M_{\odot}$ are identical, but $R / R_{\mathrm{H}}$ is different as labeled. Differences in $R / R_{\mathrm{H}}$ are equivalent to changing distance from the Sun, or planetary density, or both. In the experiments represented here, the abscissa values were changed by varying $V$, not $V_{e}$.

as Wetherill and Cox did. In all these cases from Greenzweig and Lissauer's Fig. 8 (our Fig. 5), the mass of the planet is fixed at $10^{-6}$ times the solar mass and, as with Wetherill and Cox, $e=2 i$. In each figure a particular planetary radius (expressed as a fraction of the Hill radius) was selected and held constant. Also, in each case an experiment was performed with all particles on initially circular orbits $(V=0)$. The case $V=0$ is off-scale, so we show that result with an arrow just off the right edge.

We also show the impact rates given by our analytical formulae as the solid curves on these figures. Again the regime boundaries are plotted as abrupt transitions, although gradual transition is expected according to the theory. Our theoretical results match the Monte Carlo experiments quite well in all regimes, including excellent agreement of our constant rate in Regime $C$ with the experimental rate at $V=0$.

Other Monte Carlo experiments by Greenzweig and Lissauer (their Table II) are not readily comparable with our analysis because they lie close to the transition region between random and keplerian control [See Eq. (10)]. However, these additional results of Greenzweig and Lissauer are discussed further in the next section in comparison with the results by Ida and Nakazawa.

\section{III.C. Experiments of Ida and Nakazawa}

Ida and Nakazawa (1989) performed a similar set of numerical experiments. They show impact rates (rather than the scaled quantity $F_{\mathrm{g}}$ ) as a function of $e$ and $i$, in the 


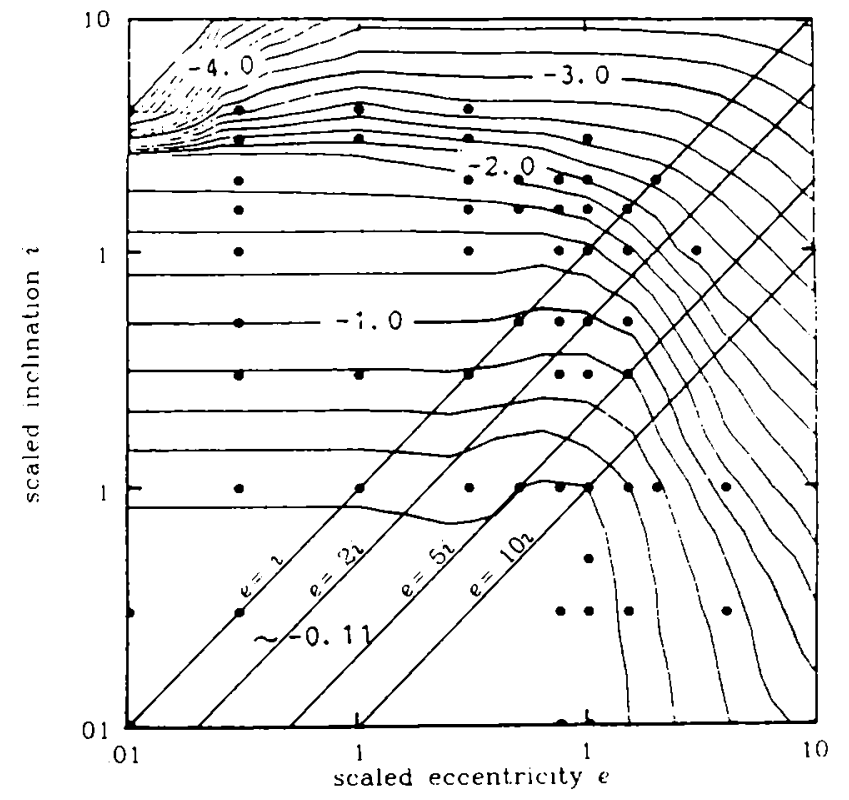

FIG. 6. Impact rates from Monte Carlo experiments of Ida and Nakazawa (1989) as a function of $e$ and $i$, shown by contours. Scaling is defined in the text. We superimpose points indicating values of $e$ and $i$ at which their experiments were actually performed. which yielded the published contours. The diagonal slices at fixed ratios ei $i$ can be used to generate displays (Fig. 7) in a format similar to Figs. 4 and 5.

contour plot in Fig. 6 (based on their Fig. 14). Here $e$ and $i$ are scaled to the size of the Hill sphere:

$$
\begin{aligned}
& \text { Scaled } e=e \frac{R_{\mathrm{H}}}{a}, \\
& \text { Scaled } i=i \frac{R_{\mathrm{H}}}{a} .
\end{aligned}
$$

In other words, for example, if the scaled $e=1$, the radial oscillation is the same size as the Hill sphere.

A couple of caveats must be noted for interpreting these results. First, these contours represent interpolation (and extrapolation) among limited data points (which we show in Fig. 6) so caution is needed in any interpretation of detailed structure (or of any structure in some regions). Second, Greenzweig and Lissauer (1990) attempted to reproduce these results with their own Monte Carlo tests and found as-yet-unexplained quantitative disagreement as great as $75 \%$ in certain parts of $(e, i)$ space. While the results in Fig. 6 must therefore be interpreted with appropriate caution, they probably represent qualitative trends reasonably well.

Slices through $(e, i)$ space along lines with constant $e / i$ yield curves in the same format as Figs. 4 and 5. Ohtsuki and Ida (1990) show such curves (reproduced here as Fig.
7) for four different values of $e / i$, each of which was apparently generated by manually transferring data from one of the diagonal slices that we show in Fig. 6. The caveats regarding Ida and Nakazawa's results, as well as additional concerns, apply to Fig. 7:

(1) Note that the "scaled velocity" is defined as $\sqrt{e^{2}+i^{2}} /\left(R_{\mathrm{H}} / a\right)$. The scaling to $R_{\mathrm{H}}$ and their choice of units with $a=n=1$ are perfectly rigorous. However, this definition is equivalent to (2) rather than the more refined form (6).

(2) Most of these diagonal slices lie between the actual data points used to generate the contours in Fig. 6, so any detailed structure in Fig. 7 is suspect.

(3) From scaled velocity values of $\sim 1$ up to about $\sim 2$, the rates shown become increasingly too low relative to the reevaluation by Greenzweig and Lissauer.

(4) The data points shown in Fig. 6 are extremely sparse for scaled $e>2$, so the curves in Fig. 7 do not really represent results of numerical experiments for "scaled velocity" greater than about 2 .

(5) We infer that the portions of their curves to the right of that value were generated from Ohtsuki and Ida's (and Ida and Nakazawa's) version of the analytic formula for impact rate in Regime $A$, with a hand-drawn transition between the experimental results and the analytical results.

(6) Their analytical results for Regime A are not precise, and are based on a hybrid formulation. While they do adopt the accurate form (A7) for the accretion rate (the same as Greenzweig and Lissauer), they use the approximate expression for the velocity (A2) in the evaluation.

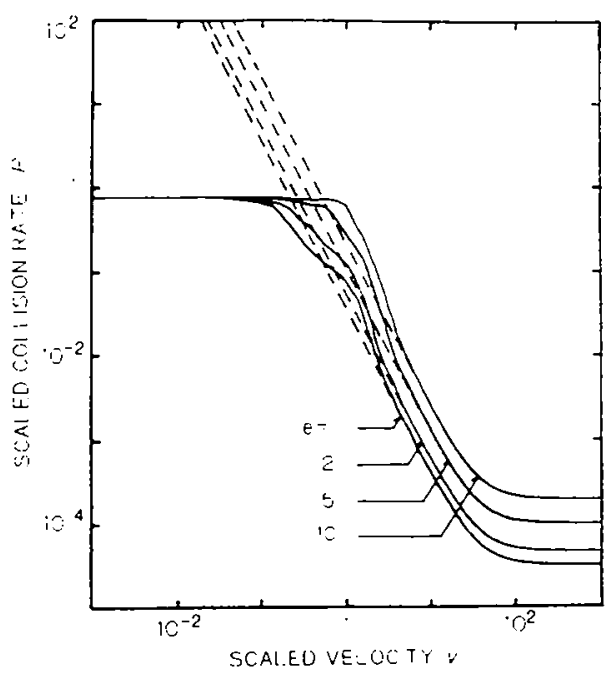

FIG. 7. Impact rates plotted by Ohtsuki and Ida (1990), based on diagonal slices through the contours in Fig. 6. Scales are defined in the text. For $V \geq 2$, results appear to be based on an approximate analytical theory, and not on numerical experiments. For $V \geq 1$, even the numerical results are suspect, according to Greenzweig and Lissauer (1990). 


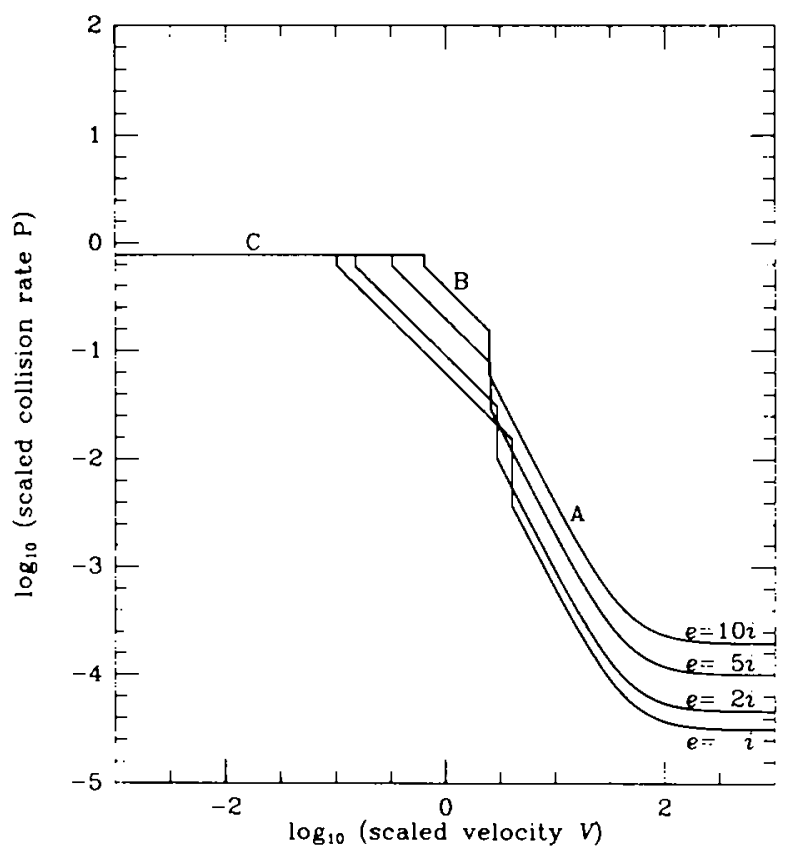

FIG. 8. Impact rates from our analytical theory for the cases plotted by Ohtsuki and Ida, to the same scale as Fig. 7. Our results are similar to the results in Fig. 7 for $V \leqslant 1$, but they diverge for larger $V$. We are thus in accord with Greenzweig and Lissauer's experimental results for the same case.

Results generated from our analytical expressions are plotted in Fig. 8 to the same scale and for the same parameters as in Fig. 7. Our results are in excellent agreement with Ohtsuki and Ida's numerical experiments where their data are credible, i.e., for scaled velocity $<1$. From scaled velocity 1 to 2 , our results become increasingly high relative to theirs; thus we are in excellent agreement with Greenzweig and Lissauer's reevaluation over this range. Further to the right in these figures, the curves of Ohtsuki and Ida are not based on numerical experiments, but rather on the relatively imprecise formulae they adopted for Regime A, so they are only qualitatively similar to our results.

As a final means of comparison, we have evaluated impact rates using our formulae over the entire $(e, i)$ space plotted by Ida and Nakazawa to produce an analytically derived version of Fig. 6 . The various analytical regimes are shown in Fig. 9. In this case, based on (10), scaled $e<2.3$ means that keplerian shear rather than random motion dominates the impact statistics. In this region, (19) gives the value of $i$ that separates Regime $B$ from the essentially two-dimensional Regime C, as shown in Fig. 9.

Note however that for $e>2.3$ (random motion dominates) it is also possible to have essentially two-dimensional behavior if $e \gg i$. This defines a fourth regime (D) that we have not discussed up to this point because it is unlikely to pertain to a real dynamical system, and be- cause construction of analytic formulae is trivial once Regime A is understood. However, it must be considered if we are to completely map the $(e, i)$ space represented by Ida and Nakazawa.

The boundary between Regimes $A$ and D occurs where the planet's gravitational diameter $2 R_{\mathrm{G}}$ becomes comparable to the thickness of the planetesimal swarm:

$$
2 R\left(1+\frac{V_{\mathrm{e}}^{2}}{V^{2}}\right)^{1 / 2}=2 a i
$$

This boundary is a curve in $(e, i)$ space because $V$ is given by (6). However, the expression can be simplified because it only applies where $i \ll e$, so $V$ is simply $\sqrt{\frac{5}{8}}$ nae. The impact rate in Regime $D$ is the two-dimensional version of (5)

$$
\text { Impact rate }=\frac{5}{8}(2 R)\left(1+\frac{V_{\mathrm{e}}^{2}}{V^{2}}\right)^{1 / 2} V \sigma
$$

Evaluation of the impact rate over $(e, i)$ space gives the results shown in Fig. 10, which reproduces the results of Ida and Nakazawa to the extent that the latter are valid. Diagonal slices through this space would give the same curves shown in Fig. 8. The only place where our analytical results significantly differ from the numerical results is in Regime B for scaled $i>2.5$, where the analytical formulae overestimate impact rates, as expected ac-

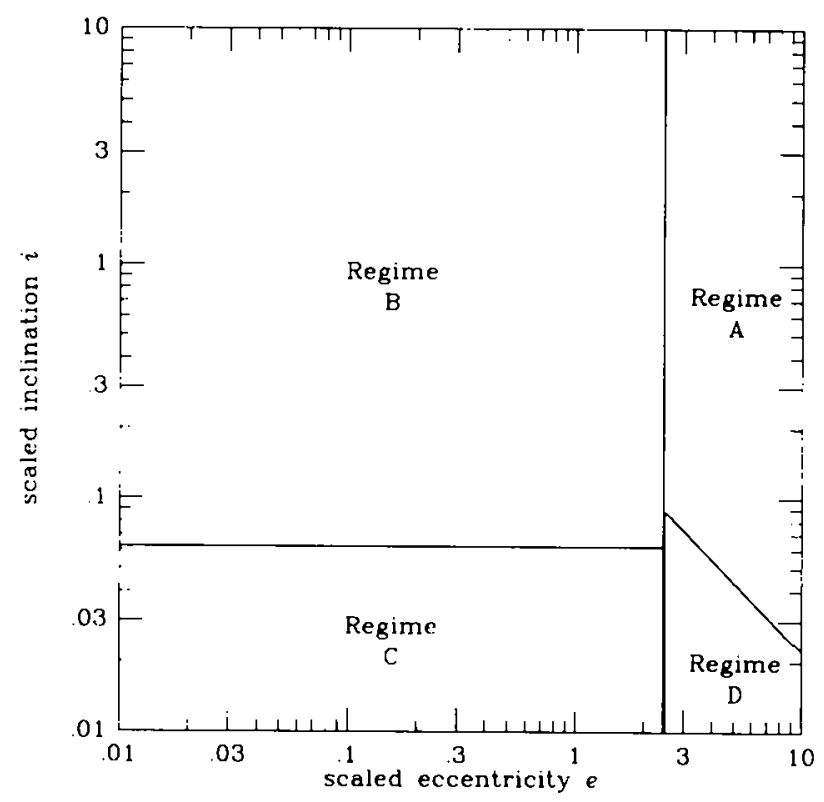

FIG. 9. The region of $(e, i)$ space mapped by Ida and Nakazawa (cf. Fig. 6), divided into the analytical regimes discussed in the text. 


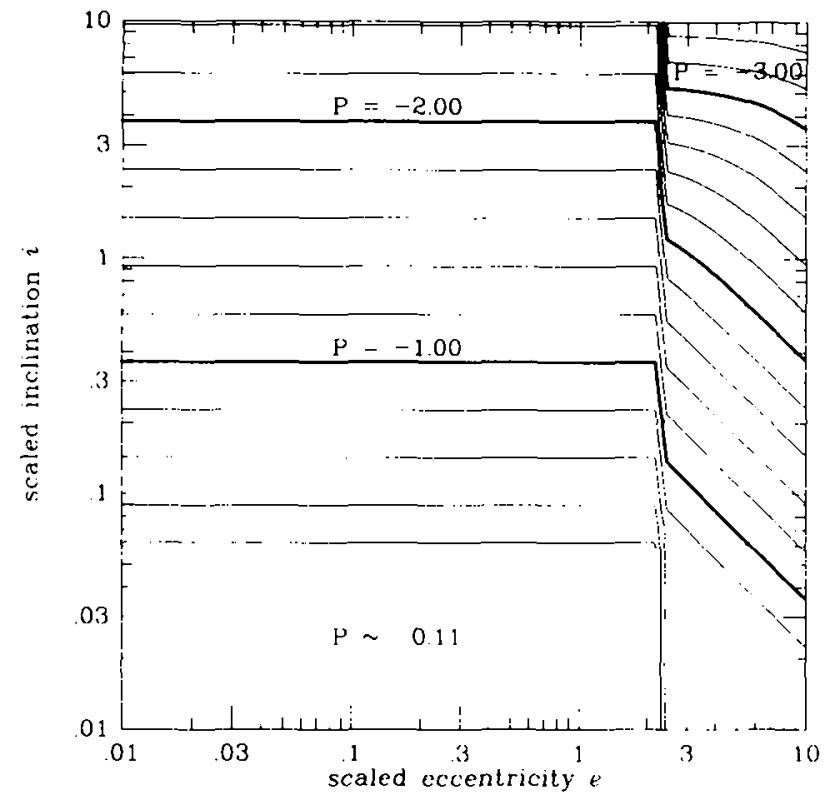

FIG. 10. Impact rates from our analytical theory, displayed as contours over the same space as mapped by Ida and Nakazawa's Monte Carlo work. (Compare with Fig. 6.) The qualitative agreement is reasonable. especially taking into account the various caveats regarding the precision of Ida and Nakazawa's results.

cording to the explanation by Ida (last paragraph of Section II.B.2).

\section{CONCLUSIONS}

The excellent agreement between our analytical theory and the numerical Monte Carlo experiments of various researchers indicates that we now understand the basic physical processes that control impact rates for a swarm of planetesimals interacting with a growing planet on a circular orbit, or for any similar system with a finite orbiting body in a swarm of test particles.

In the range of relatively large random motion that we call Regime A, impact rates are enhanced to some degree by the gravitational cross-section of the target planet. Further enhancement for smaller $V / V_{\mathrm{e}}$, which was noted in Monte Carlo experiments, is now explained in terms of the transition from a system dominated kinematically by random motion (larger $V / V_{\mathrm{c}}$ ) to one dominated by keplerian shear. For still smaller $V / V_{\mathrm{c}}$, the system becomes effectively two-dimensional and the enhancement levels off.

Understanding the random-to-keplerian transition is critical to the issue of "intermediate-stage" evolution of the protoplanetary swarm. Greenberg et al.'s (1978) simulation of the earlier stage assumed that random motion was dominant. But once a state was reached with large embryos and low random velocities (low $V / V_{\mathrm{e}}$ ), the simu- lation could not be reliably continued. The random-tokeplerian transition marks the beginning of the poorly understood intermediate stage of planet growth.

Monte Carlo results gave some indication of the change in accretion rates for smaller $V / V_{\mathrm{e}}$. Empirical parameterization of those results by including an "enhancement" factor in the impact rate can be incorporated into simulations of intermediate-stage evolution, but the generalization of experimental results from specific cases is always suspect.

Our analytic derivation gives us a physical basis for extending the generality of the Monte Carlo results, and thus will be useful for further modeling of planet growth through the stages that are still only poorly understood. We note that the analytical results reported here depend heavily on the earlier numerical work in two ways. First, the deviation of formulae in the keplerian regimes ( $B$ and C) was based on general characteristics of the flow of initially circular orbits as they approach a planet, as demonstrated by numerical integration. Second, our analytical derivations were only designed to have order-of-magnitude precision; the excellent agreement with Monte Carlo experiments gives us much greater confidence in their validity than we could have claimed without the experimental confirmation. We emphasize however that the absolute values of impact rates given by our formulae were expected to have uncertainties as great as factors of 2 or 3 . The close agreement suggests either that our adopted estimations at various stages were reasonable, or that the various uncertainties canceled one another out. More significant than absolute values is that the functional dependencies (including transitions between regimes) agree well with numerical experiments.

Thus the combination of our analytic model with the Monte Carlo results provides us with understanding and tools for further study of the planet-formation process that are much stronger than either approach could have provided alone.

\section{APPENDIX A}

The approximate equations (1), (2), and (4) can be expressed in exact form by introducing correction coefficients. In the nongravitational case [equivalent to Eq. (1)]

$$
\text { Impact rate }-K_{1} \pi R^{2}\left(\frac{V \sigma}{2 a i}\right)
$$

In (AI) we can take $V$ to be precisely

$$
V=n a \sqrt{e^{2}+i^{2}}
$$

with any necessary correction to the actual random velocity subsumed 
into $K_{1}$. Similarly, taking into account the gravitational cross-section, we have the equation [equivalent to Eq. (4)]

$$
\text { Impact rate }=K_{2} \pi R^{2}\left(1+\frac{V_{\mathrm{e}}^{2}}{V^{2}}\right)\left(\frac{V \sigma}{2 a i}\right)
$$

where again $V$ is given by (A2). The correction coefficient $K_{2}$ is different from $K_{1}$ due to the different dependence on $V$. (Here the subscript 2 serves as a reminder that the encounter assumes two-body dynamics).

Because Eqs. (1), (2), and (4) were taken to be reasonably good orderof-magnitude estimates, we expect $K_{1}$ and $K_{2}$ to be of the order of unity. In fact, as noted in Section II.A, $K_{1}$ and $K_{2}$ are often assumed to be 1 in the literature. The problem of finding more precise forms of these equations is equivalent to evaluation of the correction coefficients.

\section{Evaluation of Correction Coefficients from Öpik Theory}

A precise evaluation of the accretion rate in Regime A comes from the work of Öpik (1951). This approach considers the full range of orbital elements for planetesimals with a given $e$ and $i$ that cross the orbit of the target planet. The fraction of orbits that pass within the target area of the planet in a given time increment yields impact-rate formulae similar to (1) and (4). We note some possible shortcomings to this approach: It does suffer from some assumptions that break down for nearly tangential orbits. Also, it involves combining the average encounter velocity with the average encounter rate, rather than the more rigorous method of averaging after combination. Nevertheless, this approach gives the most well-justified analytical expressions available at present. Besides Öpik's own applications of this approach, it has been used by Wetherill (1967), Greenberg (1982), and Greenzweig and Lissauer (1990).

Greenzweig and Lissauer (1990) used that approach to obtain the expression for the nongravitational case [equivalent to Eq. (AI)],

$$
\text { Impact rate }=\frac{2 n \sigma R^{2}}{\pi} \frac{\sqrt{e^{2}+\sin ^{2} i}}{\sin i} \mathbf{E}(k),
$$

where $\mathbf{E}$ is the Complete Elliptic Integral of the 2nd Kind and

$$
k=\frac{\sqrt{3} e}{2 \sqrt{e^{2}+\sin ^{2} i}}
$$

Note that Greenzweig and Lissauer chose units such that $a=n=1$; here we display $a$ and $n$ in all equations so the dependencies are apparent. The correction coefficient $K_{1}$ is found by dividing (A4) by (AI):

$$
K_{1}=\frac{4}{\pi^{2}} \frac{\sqrt{e^{2}+\sin ^{2} i}}{\sqrt{e^{2}+i^{2}}}\left(\frac{i}{\sin i}\right) \mathbf{E}(k) .
$$

The values of $K_{1}$ as a function of $e$ and $i$ are shown in Fig. Al, which confirms that the factor is of order unity, within the narrow range 0.49 to 0.64 .

For the case of gravitationally enhanced cross-section Greenzweig and Lissauer gave the expression [equivalent to Eq. (A3)]

$$
\text { Impact rate }=\frac{2 n \sigma R^{2}}{\pi} \frac{\sqrt{e^{2}+\sin ^{2} i}}{\sin i} \mathbf{E}(k)\left(1+\frac{V_{\mathrm{e}}^{2}}{V_{\mathrm{GL}}^{2}}\right) \text {, }
$$

where $V_{\mathrm{GL}}$ is the adopted expression, based on Öpik theory, for the random velocity,

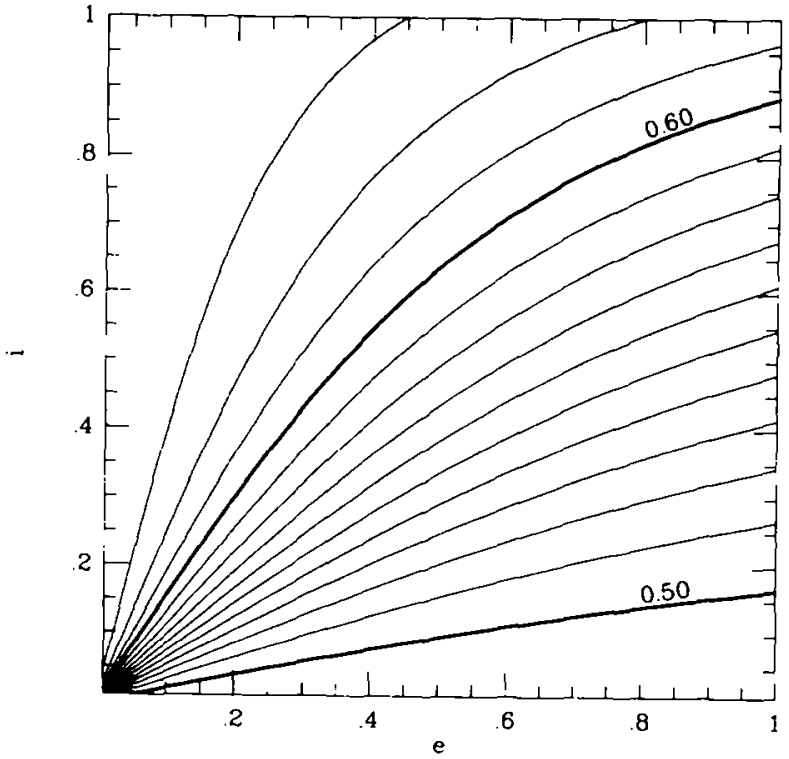

FIG. A1. Function $K_{\mathrm{t}}(e, i)$ from Eq. (A6). Contours are at increments of 0.01 . Maximum value of $K_{1}$ in this range is 0.637 at the upper left; minimum value is 0.493 at lower right.

$$
V_{\mathrm{GL}}=n a \sqrt{e^{2}+\sin ^{2} i} \sqrt{\frac{\mathbf{E}(k)}{\mathbf{K}(k)}},
$$

and $\mathbf{K}(k)$ is the Complete Elliptic Integral of the 1 st Kind. Note that although (A3) and (A7) describe the same rate, they use different expressions for the random velocity. $V_{G L}$ represents a rigorously derived value of the average relative velocity. while the expression (A2) for $V$ is an approximation for which we account with $K_{2}$ in (A3).

$K_{2}$ is evaluated by dividing (A7) by $(A 3)$ :

$$
K_{2}=K_{1}\left(\frac{1+V_{e}^{2} / n^{2} a^{2}\left(e^{2}+\sin ^{2} i\right)(\mathbf{E}(k) / \mathbf{K}(k))}{1+V_{e}^{2} / n^{2} a^{2}\left(e^{2}+i^{2}\right)}\right) .
$$

For $V \gg V_{\mathrm{e}}, K_{2}=K_{1}$. For $V \ll V_{\mathrm{e}}, K_{2} \sim 1$, as plotted in Fig. A2. (The transitional case $V_{\mathrm{e}} \sim V$ is of limited interest here.) In Figs. A1 and A2, the structure of both $K_{\mathrm{I}}$ and $K_{2}$ is fairly simple for $e$ and $i<0.2$. The more curvilinear structure for larger $i$ is due to the use of $\sin i$ rather than $i$ in Greenzweig and Lissauer's evaluation. In fact, in most applications, $e$ and $i$ are quite small, so the latter distinction is not important.

\section{Evaluation of Correction Coefficients from Safronov Theory}

Safronov (1969), as part of his landmark work in planet-formation theory, derived from statistical mechanical principles a mean random relative velocity of

$$
V_{\mathrm{Saf}}=n a \sqrt{\frac{5}{8} e^{2}+i^{2}}
$$

The coefficient 5 reflects the partition of energy among three dimensions; motion in both in-plane dimensions is controlled by $e$, with tangential motion half as fast as radial motion. Using this expression for $V$ [instead 


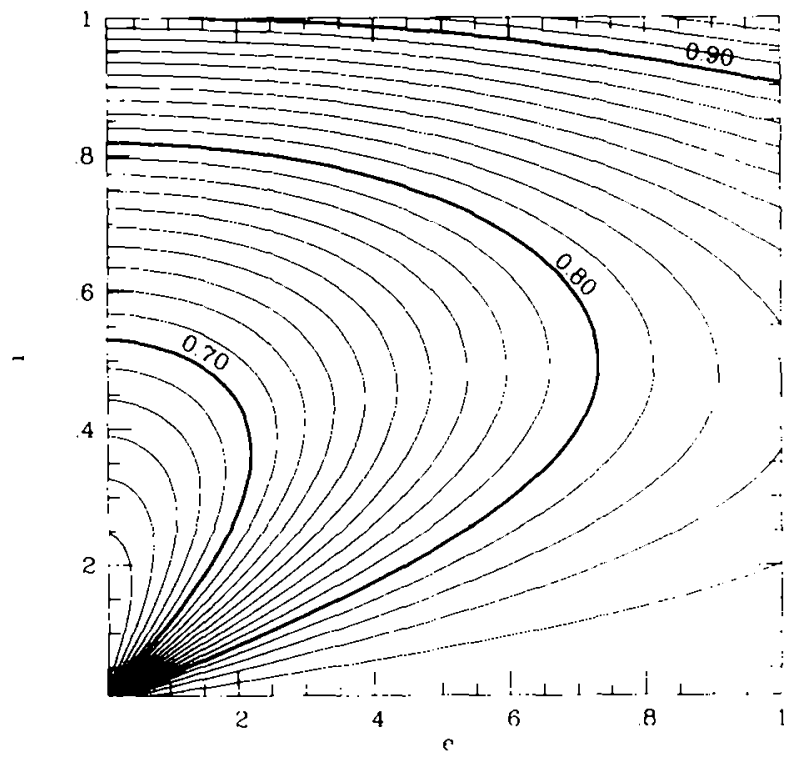

FIG. A2. Function $K_{2}(e, i)$ from Eq. (A9), with $V \ll V_{c}$. Contours are at increments of 0.01 . Maximum value of $K_{2}$ in this range is 0.940 at the upper right: minimum value is 0.640 on the left edge.

of Eq. (2)] in (1), as is sometimes done in the literature (e.g. Nishida. 1983). is equivalent to introducing a correction factor

$$
K_{1}=\frac{\sqrt{2 e^{2}+i^{2}}}{\sqrt{e^{2}+i^{2}}}
$$

This function is plotted in Fig. A3. Comparison of Fig. A3 with Fig. Al shows that these two functions for $K_{1}$ are practically identical for $e^{\prime}$ and

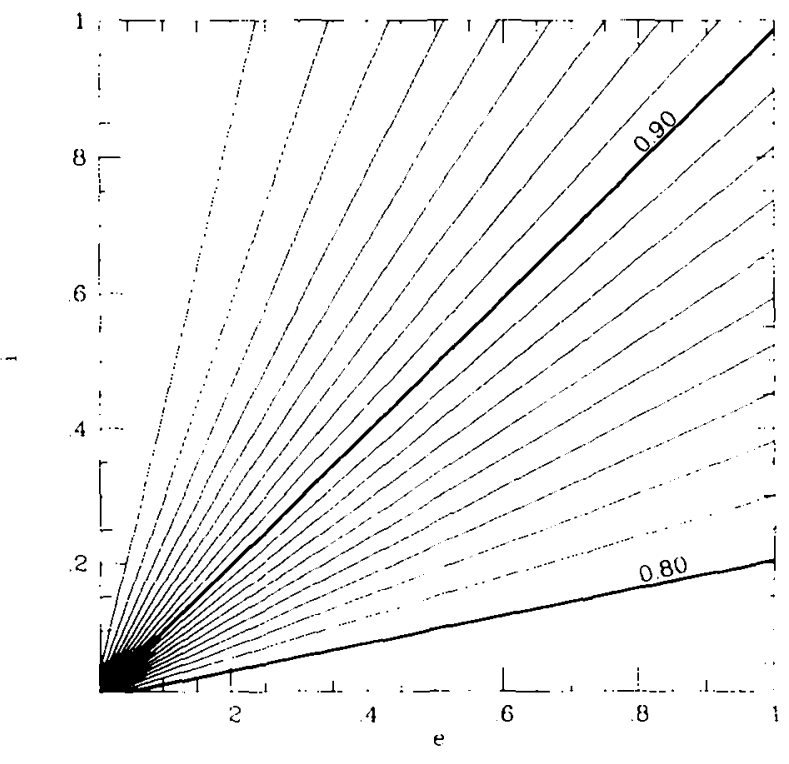

FIG. A3. Function $K_{1}(e, i)$ from Eq. (A11). Contours are at incre ments of 0.01 . Maximum value of $\boldsymbol{K}_{1}$ in this range is 1.000 at left; minimum value is 0.791 at lower right.

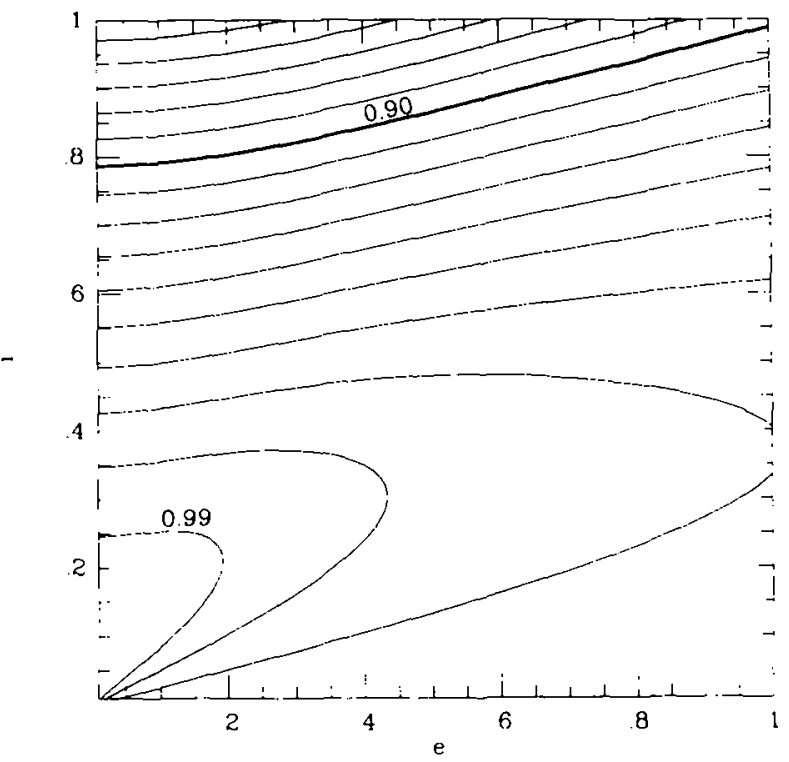

FIG. A4. Ratio of mean random velocity adopted by Greenzweig and Lissauer (A8) to that adopted by Safronov (A10). Contours are at increments of 0.01 . Maximum value of ratio in this range is 0.9997 at lower left: minimum value is 0.841 at upper left. Note the excellent agreement of the two formulations, especially for realistic (small) $e$ and $i$.

$i<0.5$ except for a factor of $\frac{1}{4}$. This observation allows us to write the equation for the nongravitational case in the very simple form

$$
\text { Impact rate }=\frac{5}{8} \pi R^{2}\left(\frac{V_{\mathrm{Sar}}(T}{2 a i}\right)
$$

to obtain an expression very close (within $1 \%$ over the values $c<0.5$ and $i<0.5$ ) to the expression adopted by Greenzweig and Lissauer (A4).

The target's gravitational cross-section is taken into account, as before. by multiplying by $1-V_{\mathrm{e}}^{2} / V^{2}$. In fact. $V_{\mathrm{Saf}}$ is within a few percent of $v_{i j}$ as shown in Fig. A4. Thus we may adopt the general equation

$$
\text { Impact rate }-\frac{5}{8} \pi R^{2}\left(1+\frac{V_{c}^{2}}{V^{2}}\right)\left(\frac{V \sigma}{2 a i}\right)
$$

(A13)

where $V=v_{\text {Sar }}$. This simple form is reasonably accurate throughout Regime $A$ and retains the same simple functional form as derived by the "particle-in-a-box." or "gas-dynamical." physical model [Eq. (4)].

\section{APPENDIX B}

\section{Ratio of Planet Radius to Hill Sphere Radius}

$$
\frac{R}{R_{\mathrm{H}}}-\frac{R}{a\left(\frac{M_{\mathrm{p}}}{3 M}\right)^{1.9}}=\left(\frac{R}{a}\right)\left(\frac{3 \rho}{D_{\mathrm{p}}}\right)^{1.3} .
$$

Thus, unless the orbit of the planet is near the surface of the Sun, or the planet is far less dense than the Sun. 


$$
R \ll R_{\mathrm{H}}
$$

As an example, for a rocky planet with $\rho=3 \mathrm{~g} \mathrm{~cm}^{-3}$ near $1 \mathrm{AU}, R_{\mathrm{H}} \approx$ $200 R$.

Relative Size of Gravitational Radius in Regime B

$$
\frac{V_{\mathrm{c}}}{V_{\mathrm{T}}}=\frac{\left(\frac{2 G M_{\mathrm{p}}}{R}\right)^{1 / 2}}{1 . \ln \Delta a} \approx \frac{\left(\frac{2 G M_{\bigcirc}}{R}\right)^{1 / 2}\left(\frac{M_{\mathrm{p}}}{M_{\odot}}\right)^{1 / 2}}{2 n a\left(\frac{M_{\mathrm{p}}}{M_{\mathrm{C}}}\right)^{1 / 3}}
$$

Using Kepler's third law and the definition (8) of $R_{\mathrm{H}}$.

$$
\left(\frac{V_{\mathrm{e}}}{V_{\mathrm{T}}}\right)^{2} \sim 0.8\left(\frac{R_{\mathrm{H}}}{R}\right) \gg 1
$$

From (15), the gravitational radius is

$$
R_{\mathrm{G}}^{2}=R^{2}\left(1+0.8 \frac{R_{\mathrm{H}}}{R}\right)
$$

Using (B2), Eq. (B5) becomes

$$
R_{\mathrm{G}}^{2}=0.8 R R_{\mathrm{H}}
$$

Thus

$$
R \ll R_{\mathrm{G}} \ll R_{\mathrm{H}} .
$$

\section{ACKNOWLEDGMENTS}

We thank R. Kolvoord and J. Lissauer for helpful comments and discussions. Dr. Shigeru Ida provided an extremely constructive referee's report. This work was supported by NASA's Planetary Geology and Geophysics Program, Grant NAGW-1029.

\section{REFERENCES}

Burns, J. A., R. A. Kolvoord, and D. P. Hamilton, 1989. An assessment of potential hazards to the Cassini Spacecraft from debris along satellite orbits. In Cassini Mission Announcement of Opportunity, OSSA-1-89. JPL PD 699-11, Vol. 13, pp. 6-1-6-20.

DANBY, J. M. A. 1988. Fundamentals of Celestial Mechanic.s, 2nd Ed., Willmann-Bell, Inc., Richmond.

Dermott, S. F., AND C. D. Murray 1981. The dynamics of tadpole and horseshoe orbits I: Theory. Icarus 48, 1-11.

Goldreich, P., and S. TRfmaine 1982. The dynamics of planetary rings. Annu. Rev. Astron. Astrophys. 20, 249-283.

Greenberg, R., J. F. Wacker. W. K. Hartmann, and C. R. ChapMAN 1978a. Planetesimals to planets: Numerical simulations of collisional evolution. Icarus 35, 1-26.
Greenberg, R., W. K. Hartmann, C. R. Chapman, and J. F. WACKER 1978b. The accretion of planets from planetesimals. In Protostars and Planets (T. Gehrels, Ed.), pp. 599-624. Univ. of Arizona Press, Tucson.

GreEnBERG, R. 1982. Orbital interactions: A new geometrical formalism. Astrophys. J. 87, 184-195.

Greenberg, R., A. Carusi, and G. B. Valsecchi 1988a. Outcomes of planetary close encounters: A systematic comparison of methodologies. Icarus 75, 1-29.

Greenberg, R., G. B. Valsecchi, and A. Carus1 1988b. Planetesimal collision rates: Transition from random to keplerian approaches. $\mathrm{Lu}$ nar Planet. Sci. XIX, 425-426.

Greenberg, R. 1989. Planetary accretion. In Origin and Evolution of Planetary Atmospheres (S. K. Atreya, J. B. Pollack, and M. S. Matthews, Eds.) pp. 137-164. Univ. of Arizona Press, Tucson.

Greenzweig, Y. and J. J. Lissauer 1990. Accretion rates of protoplanets. Icarus 87, 40-77.

HILL, G. W. 1878. Researches in the lunar history. Am. J. Math. 1, 5-26, 129-147, 245-260.

IDA. S., AND K. S. NAKAZAWA 1989. Collisional probability of planetesimals revolving in the solar gravitation. III. Astron. Astrophys. 220, 293-300.

Nishida. S. 1983. Collisional processes of the planetesimals with a protoplanet under the gravity of the proto-Sun. Prog. Theor. Phys. 70, 93-105.

OHTSUKI, K., AND S. IDA 1990 . Runaway growth with collision rate in the solar gravitational field. Icarus 85, 499-511.

ÖPIK, E. J. 1951. Collisional probabilities with the planets and the distribution of interplanetary matter. Proc. $R$. Irish Acad. A54, 165-199. [See also Öpik, E. J. 1976. Interplanetary Encounters, Elsevier.]

Petit, J.-M., ANd M. Hfinon 1986. Satellite encounters. Icarus 66, 536-555.

Roy, A. E. 1988. Orbital Motion. 3rd. ed., Hilger, Bristol and Philadelphia.

SAFronov, V. S. 1969. Evolution of the Protoplanetary Cloud and Formation of the Earth and the Planets. Nauka Press, Moscow. NASA TTF-677, 1972.

StEWART, G. R., AND G. W. Wetheril. 1988. Evolution of planetesimal velocities. Icarus 74, 542-553.

Wetheritl, G. W. 1967. Collisions in the asteroid belt. J. Geophys. Res. 72, 2429-2444.

WETHERII.L, G. W. 1980. Numerical calculations relevant to the accumulation of the terrestrial planets. In The Continental Crust and Its Mineral Deposits (D. W. Strangway, Ed.). pp. 3-24. Geol. Assoc. of Canada Special Paper 20

WetheriLl, G. W., AND L. P. Cox 1984. The range of validity of the two-body approximation in models of terrestrial planet accumulation I: Gravitational perturbations. Icarus 60, 40-55.

WETHERILL, G. W., AND L. P. Cox 1985. The range of validity of the two-body approximation in models of terrestrial planet accumulation II: Gravitational cross-sections and runaway accretion. Icarus 63 , $290-303$.

Wetheril.l, G. W., and G. R. Stewart 1989. Accumulation of a swarm of small planetesimals. Icarus $77,33()-357$. 